\title{
Trusted information project platform based on blockchain for sharing strategy
}

\author{
Eunhee Lee $^{1}\left[{ }^{\mathbb{D}} \cdot\right.$ Yongik Yoon $^{1}$
}

Received: 5 October 2018 / Accepted: 1 August 2019 / Published online: 21 August 2019

(c) The Author(s) 2019

\begin{abstract}
South Korea invests a budget of trillions in national R\&D projects every year, and has achieved excellent performance doing so each year. However, since the projects are planned, evaluated, and managed by different departments and institutions, duplicate planning and submission leads to insufficient sharing of research results. Currently, the National Technology Information Service (NTIS) inspects project duplication based on keywords, which leads to duplicate planning among departments and closed management of research results. Since the NTIS builds in centralized systems, the inspection systems supports one-way management for duplication checking and information sharing. Therefore, we propose a new platform, called the Trusted Information Project Platform (TIP-Platform), for easily checking for project duplication, sharing research results, and updating research results. TIP-Platform adopts a new concept for user authority setting, the distributed ledger structure, transaction structure, and service. For the adaption, the TIP-Platform uses blockchain technology that performs recording and management via blocks by distributing the right to record and managing transactions. This platform makes it easy for anyone to view and use project-related information such as research results and duplication review. In this paper, we describe how the TIP-Platform can achieve excellent research results through information sharing of a project. This platform needs to be based on trust, because it shares information and continually updates information.
\end{abstract}

Keywords Distributed system · TIP-Platform · Sharing project information · Sharing duplication project information · Distributed ledger $\cdot$ Transaction structure $\cdot$ Blockchain

\section{Introduction}

According to the World Economic Forum (WEF), blockchain technology is expected to account for $10 \%$ of global GDP by 2025 . The United Nations Future Report 2050 and the top 10 promising technologies of the global IT market research organization continue to include blockchain technology. Blockchain technology has generally been focused on financial services such as bitcoin, but it is gradually expanding to various services. In this paper, blockchain technology is applied to national $R \& D$ projects in order to derive new services.

Yongik Yoon

yiyoon@sm.ac.kr

Eunhee Lee

eunhee@iitp.kr

1 School of IT Engineering, Sookmyung Women's University, Seoul 04310, Korea
Currently, in Korea, national R\&D projects are planned, evaluated, and managed by numerous departments and institutions. This means that each organization deals with different planning, evaluation, and management tasks. In addition, each organization operates a project planning, evaluation, and management system, so the project performer must register the results of the project according to the type of NTIS system. This leads to issues of duplicated planning and submission, as well as insufficient sharing of research results. Information for $\mathrm{R} \& \mathrm{D}$ projects is also important when proposing a national $R \& D$ project and sharing the results of the projects performed. With project information sharing, better projects can be proposed and duplication can be avoided. It is possible to reduce the cases of duplication after the proposal. It can also reduce cases where project proposals are invalidated. In addition, in the process of research, the scope of sharing is set up, and the projects are organized as private within the scope of the same project group. Information that needs to be shared as a whole, such as the information 
and the results of the project, is constructed and shared in a public manner.

This study aims to use blockchain technology for sharing information of national R\&D projects based on the characteristics of data openness, security, stability, and efficiency. In order to easily share the contents of the national ICT R\&D project, we propose a platform called the Trusted Information Project Platform (TIP-Platform). This platform is based on blockchain and can achieve excellent research results through information sharing of project.

In Sect. 2, we describe the status of current national $\mathrm{R} \& \mathrm{D}$ project management, trends in platforms based on blockchain technology and the overall contents of the TIPPlatform. In Sect. 3, we define the structure and function of the TIP-Platform and the service structure. In Sect. 4, we describe TIP-Platform's service process and protocol definition. Finally, we describe the ledger and transaction structure.

\section{Related work}

\subsection{Project management method}

Over a 17-year period, 35 ministries conducted 61,280 projects [Analysis of National R\&D Project Survey Results for 2017 (2018)]. The National R\&D project research institute conducts surveys on project results and project information. The survey target information was conducted on two project items (business purpose and project details), 12 inputs (research cost, technical classification, research personnel, etc.), and six project results (paper, patent, technical fees, etc.). This means that about $1,225,600$ data were ultimately generated based on the number of projects performed and the project results. Currently, national $R \& D$ projects are carried out by about 35 Ministries, and it is difficult to share information between them because they store data on national $R \& D$ project information by ministries. It is necessary to share the information of national R\&D project information and project results scattered by each ministry. For the efficient sharing of information for R\&D projects, we propose a TIP-Platform to share information and research achievements of national R\&D projects.

\subsection{Block chain based platform trend}

In recent years, blockchain technology has begun spreading into various industries, including the financial industry (Kwak 2017; Tapscott and Tapscott 2017). In countries such as the US, Japan, and China, blockchain has been adapted in various industries. The IDC (International Data Corporation) predicted that the financial industry can save up to approximately 20 billion dollars by 2022 with blockchain technology, and McKinsey expects that the financial cost savings related to client database management and security will reach 23 trillion South Korean Won per year if blockchain technology is applied in financial systems (Lim et al. 2018). According to a global market research institution, the market size of blockchain technology is expected to reach 3.1 billion dollars in the world market and 16.9 billion South Korean Won in the domestic market by 2021, and 4.1 billion dollars for in world market and 41 billion South Korean Won in the domestic market by 2023 (Lim et al. 2018). This shows that the blockchain market is expected to grow rapidly. Coinplug, a domestic company, developed a platform called "FidoLedger" to which private blockchain technology was applied, and is providing various services to financial firms and public institutions using data distribution contract technology with this platform [www.bloter.net/archives (2018)]. Enigma, developed by MIT, is a blockchain platform that enables joint data operation by participants while keeping data completely private. As it is a platform enabling data operation while keeping the contents of the data private, blockchain technology allows for the safe storage of data (2018). BigchainDB provides a database that can expand and retrieve while maintaining the characteristics of the existing blockchain. BigchainDB manages two databases: one of them stores the transaction information sent by clients while the other adds a block containing the transaction information onto a chain and stores it. Consensus is required between database nodes for BigchainDB to put transaction information into a block, and the BigchainDB Consensus Algorithm was developed in a way similar to the existing blockchain proof of stake (POS) (2018). Furthermore, IBM developed a Bluemix platform based on Hyperledger Fabric, an opensource platform. Bluemix provides various blockchain services ranging from the financial field to the music industry in a cloud environment. Microsoft also provides cloud-based blockchain services (2018) (Table 1).

\section{TIP-platform}

Blockchain is a technology in which the individuals of a distributed network can share data and continuously perform synchronization without a central server or central administrator (BitFury Group 2015; Kakavand 2017; Swan 2015). As such, this study attempts to utilize blockchain technology for sharing projects, project outputs, and checking duplication. Duplication check means duplicating project contents and subject and submitting the same research output as another project output. R\&D projects of National information communication technology (ICT) have been performed by different departments and institutions. Therefore, it is difficult to review project duplication during project proposition as the management 
Table 1 Platform classification

\begin{tabular}{llll}
\hline Classification & Type & Storage & The main content \\
\hline FidoLedger & Private blockchain & Cloud services & Data distribution technology \\
Enigma & Public blockchain & Cloud services & Data safety security \\
BigchainDB & Private blockchain & Cloud services & Extensible and searchable \\
Bluemix & Public blockchain & Cloud services & Various services such as \\
& & & finance, music, etc \\
\hline
\end{tabular}

of project performance is performed by each department or institution. Currently, NTIS inspects project duplication based on the following keywords: project name, principal researcher name, project management institution name, research objective, research contents, expectation effectiveness, korean keywords, and english keywords. The principal researcher name and project management institution name are optional, and their input are determined by the project performer; however, the other items are required. The research objective and research contents, which are the most important items for duplication review, must be prepared using 50-2000 characters based on the Korean language, and around five keywords in both Korean and English must be prepared. In addition, project duplication review is usually only mandatory in the stages of project proposition and project selection. Such reviews are difficult to perform in the project execution and evaluation stages (2016; Jun 2018). It is difficult to inspect project duplication in the present situation. This is because the amount of data entered for duplicate duplication is insufficient. The performances of the projects are also managed by NTIS. The representative performances of research results include papers, patents, royalties, and commercialization, which are registered and managed in the NTIS system. As this information can also only be accessed by dedicated institution personnel and people related to projects, it is urgently necessary to release such information.

In order to resolve the issue of duplication checking and easily checking the project results, we propose a new platform, called the Trusted Information Project Platform (TIP-Platform). This platform makes it easy for anyone to view and use project-related information. The platform allows for project managers to update related information. Through this process, platform information can develop into massive and useful information, and users can achieve excellent research results using relevant information. Therefore, the purpose of this study is to share the contents of the national ICT R\&D project, the research results, and a duplication review. We propose a block-chain-based platform that can achieve excellent research results through information sharing of projects.
This platform needs to be based on trust because it shares information and continually updates information.

\subsection{Structure of TIP platform}

In the TIP-Platform for sharing national $R \& D$ projects, TIP-Platform users can be broadly divided into users and project management departments. As shown in Fig. 1, the user can obtain project information from the TIP-Platform. The users are categorized in two types: performers and managers. The manager has the authority to generate the blockchain and is the so-called SuperUser. The performer is responsible for $\mathrm{R} \& \mathrm{D}$ projects. Based on the information of the TIP-Platform, it is possible to check for duplication of projects proposed by users and the existing projects when suggesting projects. The results of the projects can also be reviewed for duplication with other projects. The blockchain-based TIP-Platform can also be used to share information about national R\&D projects to users. The SuperUsers of project management in departments register their project information with the TIP-Platform to share the project progress information. Based on this information, users can issue new $R \& D$ projects through duplication review and sharing review. The information is then shared again with the department users. This not only serves to update new information but also to share existing information. General users can obtain information on the project from the TIP-Platform. Thus, it can request updates of relevant information so that information on national R\&D projects can continuously be updated. The continuous update request and update of the general user and department user are effective for updating the TIP-Platform information. Requests and updates of information based on trust between the user and the platform. We also support key processes in the platform process so that information can be updated based on trust (Fig. 2).

Figure 3 shows a structural diagram for the proposed TIPPlatform model to be constructed to support a trust-based project sharing service (Singh and Chatterjee 2018, 2019; Kim et al. 2006). The TIP-Platform model classifies into six structures: 
Fig. 1 NTIS-based project information sharing method
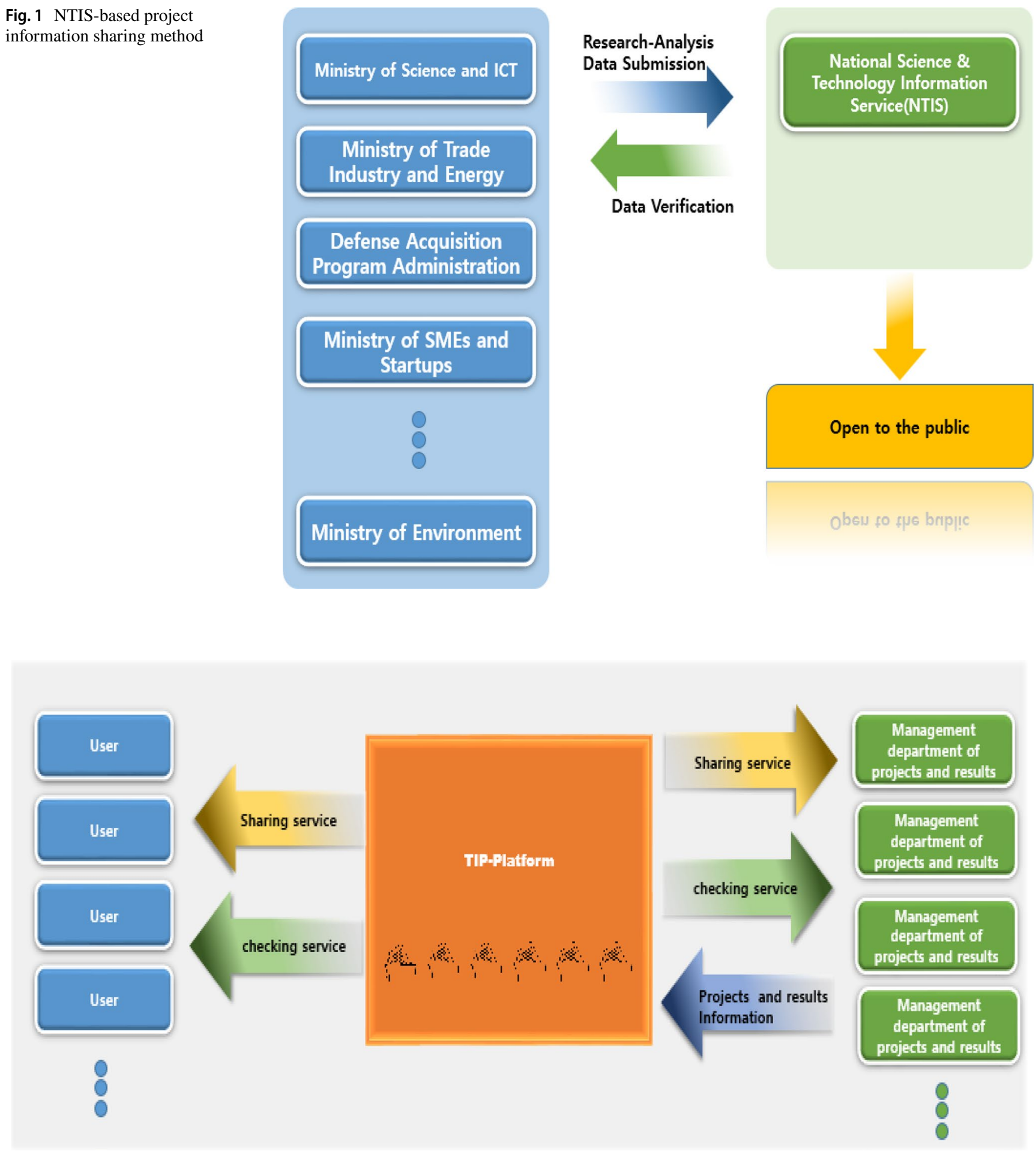

Fig. 2 Proposed TIP-platform

- Membership Manager (MM) MM handles the user authentication key granted according to the user authentication step. Permission for membership is granted according to the user's authentication, so it is possible to implement trust-based function.

- Block Mining Manager (BMM) BMM checks for duplication by reading project information. BMM steps to 
Fig. 3 Structure of TIP-platform
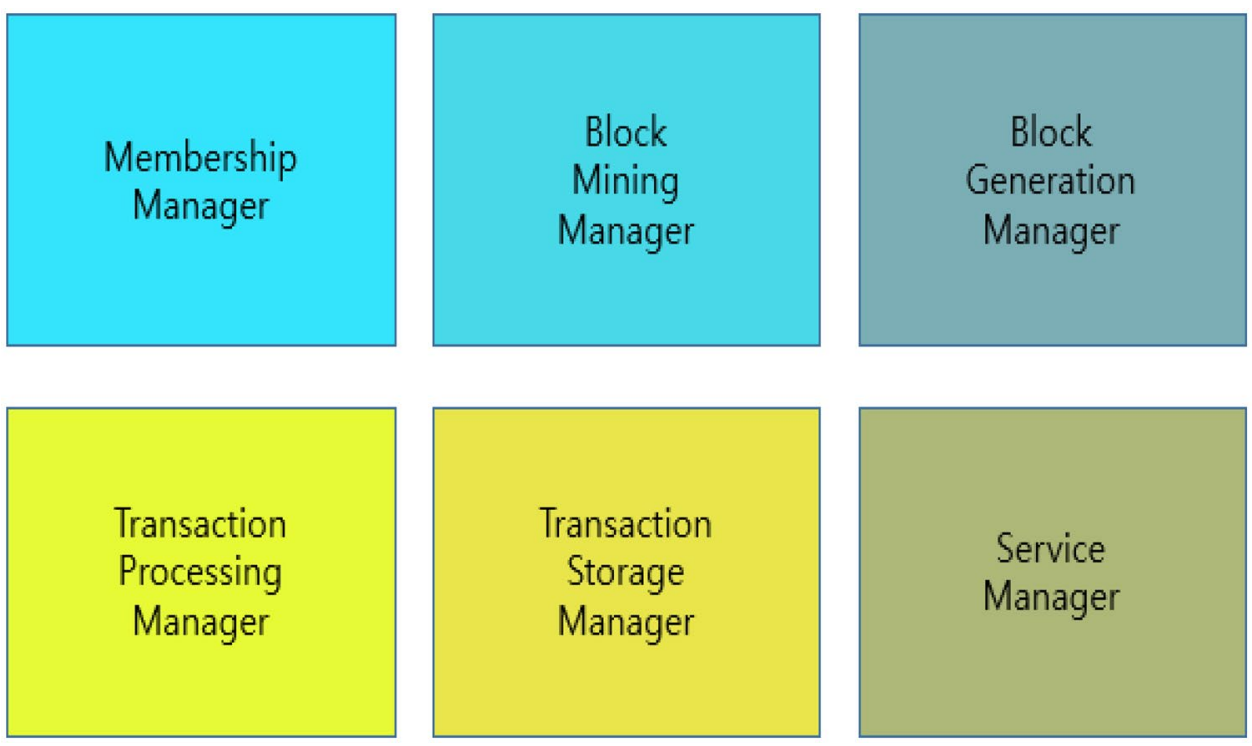

mining blocks for block generation based on the results of project duplication review.

- Block Generation Manager (BGM) BGM generates new blocks, like a ledger, including the steps of updating existing information and creating new information.

- Transaction Processing Manager (TPM) TPM configures transactions differently, including sharing project information and reviewing duplication. Transaction configuration and management comprises the model of the transaction differentially depending on the situation.

- Transaction Storage Manager (TSM) TSM categorizes according to the functions of ledger and uses the Mercury

to store and manage memory with consideration of the memory management and storage aspects.

- Service Manager (SM) SM categorizes services according to their function and performs services according to user needs.

\subsection{Service architecture of TIP-platform}

We propose a new platform for reviewing project duplication, sharing research results, and checking for duplication of research results using blockchain technology. The TIPPlatform for the sharing of project information is proposed

Fig. 4 Layer architecture for service of TIP-platform
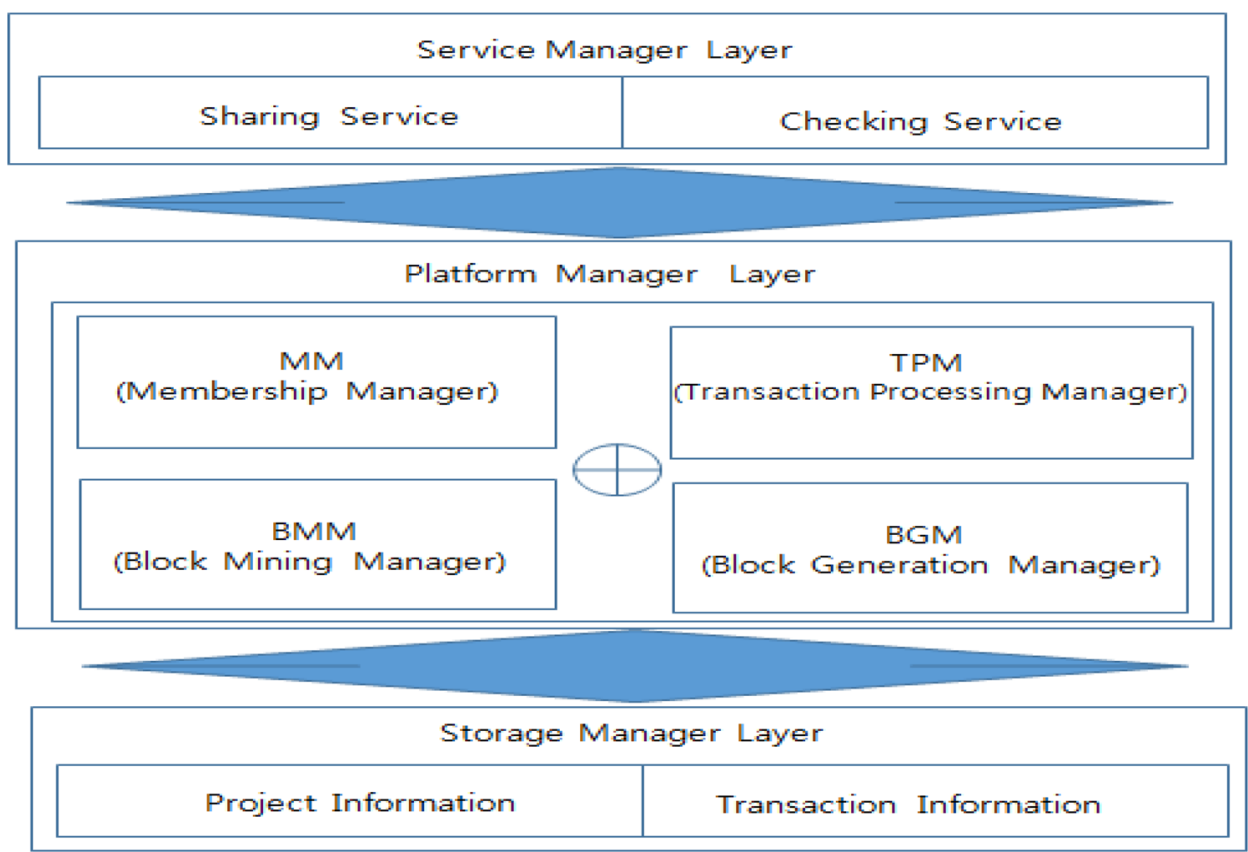
as shown in Fig. 4. The TIP-Platform has three layers as follows: service manager layer, platform manager layer, and storage manager layer.

In the service manager layer, the Service Manager provides the permission and authoring key for the services that users want. The service types for a Service Manager can be divided as follows: sharing projects progression information, checking for duplication project information, and sharing project results. The Service Manager cooperates with the membership manager in the platform manager layer in order to allow for the sharing of national R\&D projects and smooth exchange of information among users. This seamless sharing of information is closely related to the excellent performances of projects.

The platform manager layer consists of four managers as follows: the membership manager, blockchain mining manager, blockchain generation manager, and transaction manager. The membership manager specifies the user's authority and utilizes the TIP-Platform according to authority. Users who want to access this TIP-Platform can access the system by logging in. The system is largely accessed by three steps: login, personal proof, and authorization. There are three user roles depending on the system access process. The following describes the process of system access authorization.

- User Be able to simply read and review the data on the TIP-Platform.

- Performer Be able to read and write the related project information, but only reading is possible for most of the data, and proposed blocks may be created.
- SuperUser Be able to read and write all the data, proposed blocks may be created and approved.

BMM means the block mining manager. Here, an R\&D project means a block. The project has papers, patents, and products that are issued during the project of performance. $\mathrm{BMM}$ is a process of finding new project information for updating project information and updating duplicate project information. The BMM process belongs to the process of finding information.

TPM checks transactions processing for service and issues the type of ledger for the transaction. While verifying information, TPM can issue ledgers if it suspects duplication projects or duplication outcomes. In order to raise the duplication issue, TPM should issue the ledger for project progress. The ledger information for duplication issue can only be requested to be generated if the ledger including the project number, duplication keyword, and related project contents is created. When a block generation is requested, the SuperUser can accept or reject a block generation by reviewing duplication through agreement among SuperUsers. The generated blocks are available to all users for project information verification, duplication review, and verification of research results.

BGM refers to the block generation manager. BGM generates the ledger for block that includes the progress and results for projects. When there is a transaction for checking and sharing service issues, BGM should generate the ledger. A block is only created with the approval of the SuperUser. The blockchain stores projects information and creates new project information.

Fig. 5 TIP-platform process

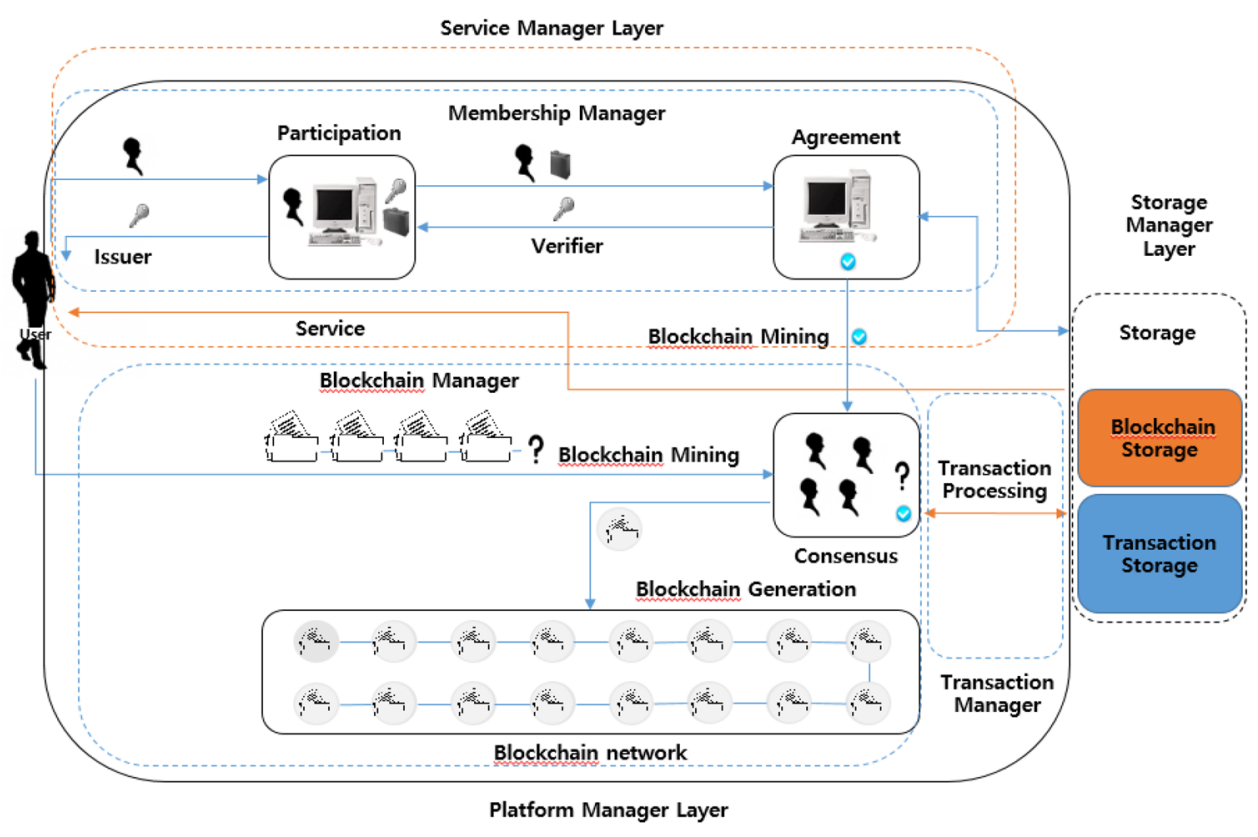


The storage manager stores project information and transaction information. For the efficient management and security of memory, we use the SHA256 hash function and the Mercury to manage the memory (Bitcoinwiki 2018). Users request for the project information to be update continuously.

\section{Implementation of TIPP}

\subsection{Process of TIPP}

Figure 5 shows how the user accesses the TIP-Platform and uses the project results and project information. Users who want to access the TIP-Platform first log in and connect to the system. According to the login information, the user can obtain the usage authority of the TIP-Platform. According to the authority of the user, it can be classified into three categories. Each user has different authority to access information and a different role to perform according to their authority. On the TIP-Platform, there are four stages. The first determines the state of the user by creating the user's identity. At this stage, the user obtains the key according to the authority. This step is called the Membership stage. The second step involves creating a distributed ledger. The user is able to access project information and the project result information. Users can propose block generation for the review of project duplication. However, both the performer and SuperUser issue block generations for transactions. This step is the Transactions stage. The third step obtains a consensus from blockchain networks to create the block. If there are service issues, an agreement is made regarding to whether or not to create the block. If the block generation proofs, the SuperUser generates the blocks for the service requests as follows: duplication check, information sharing, and progress results update. This step is called the Blockchain stage. This stage can be used by any user who is viewing information. When you update new information, you can easily share and use that information by suggesting the project, then utilizing or managing the project. The fourth step is to store the updated information. The storage steps involve not only updating project information but also storing transaction information. Through this process, it is possible to find excellent national ICT R\&D projects and create excellent project results, as well as prevent duplicate project support by sharing project information.

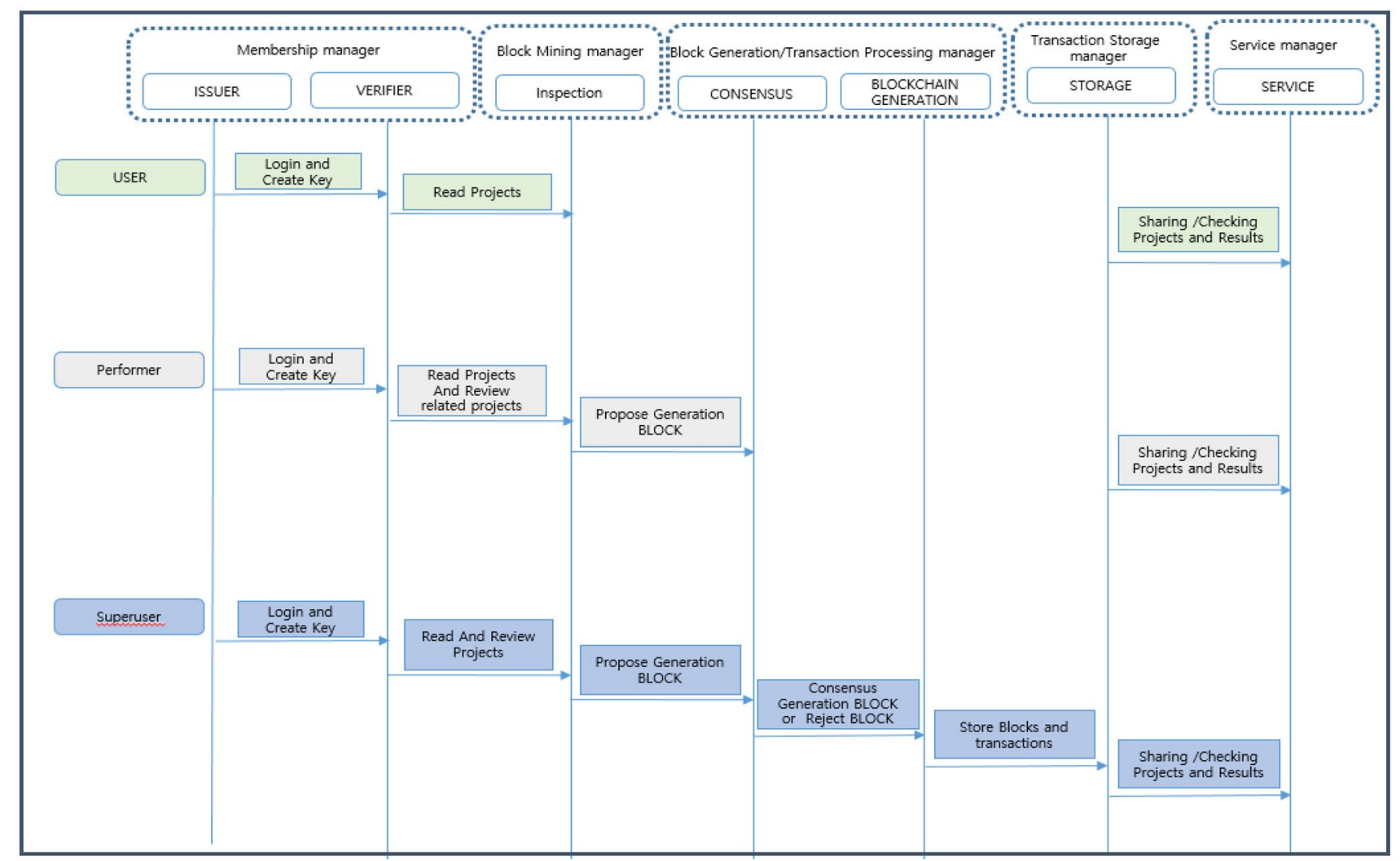

Fig. 6 TIP-platform protocol 


\subsection{Internal protocol of TIPP}

Figure 6 shows the protocol that the user accesses and the TIP-Platform information that it utilizes. The user accesses information according to each authority, we form new blocks and update information. This TIP-Platform will share and update information as shown in Fig. 6 in the order of ISSUER-VERIFIER-INSPECTION-CONSENSUStheir ID, the administrator obtains permission to access the TIP-Platform. At this time, the user receives a unique KEY.

- VERIFIER The user's authority level depends on the user's key value. We can create information according to the user's authority level.

The steps ISSUER and VERIFIER are called the membership manager stage.

- INSPECTION INSPECTION steps are part of the block mining manager as a process for reading and reviewing project information. Users suggest the creation of project information at this stage.

- CONSENSUS In order to suggest the creation of project information, users first create a ledger. The transition is based on the ledger. SuperUsers can agree to create project information or SuperUsers can decline to create project information.

- BLOCKCHAIN GENERATION SuperUsers decide to create new project information. The block is where the block generation decided to create as a blockchain. The generated blockchain is update with the new information of the project.

The CONSENSUS and BLOCKCHAIN GENERATION stages belong to the block generation manager and the transaction processing manager stage. Transactions are made through the creation of the ledger for the block generation.

- STORAGE The STORAGE stage stores new project information and transaction process information based on the cloud computer. BLOCKCHAIN GENERATION-STORAGE-SERVICE.

- ISSUER When the user logs in to the TIP-Platform with

- SERVICE The service stage shares project information and project result information at the request of users.

\subsection{BC in TIPP}

\subsubsection{Generation of BC}

For sharing the project information, the ledgers are constructed with the structure as shown in Fig. 7. The information constructed in the distributed ledger includes the project identification number, classification name, project name, keywords, project summary, project progress summary, and project results, which are key elements for project duplication review. Here, the project progress summary is the project performance information as follows: project research period, performance institution, project fund, evaluation, and progress inspection details. With the information in the ledger, we can enable sharing and duplication review of the project information.

Table 2 defines the ledger per user. The roles are determined according to the authority of each user, so the content of the ledger also depends on the role (Lee and Yoon 2019). The user is only authorized to read the information and can only submit comments if user finds a duplicate project.

Since the Performer is the user who performs the projects, the ledger constructed to reflect the changes made in the project's performance.

Table 2 Contents of Ledger

\begin{tabular}{ll}
\hline User & content \\
\hline Performer & $\begin{array}{c}\text { Enter project change history (name of project, } \\
\text { name of project leader, name of affiliation, } \\
\text { affiliation information, project change content) } \\
\text { Enter information when duplicate projects } \\
\text { are found (name of project, name of project } \\
\text { leader, name of affiliation, affiliation informa- } \\
\text { tion, project duplicate content) } \\
\text { Block creation (name of project, name of } \\
\text { project leader, name of affiliation, block } \\
\text { information) } \\
\text { The contents of the project and the duplication } \\
\text { of the research result (name of project, name } \\
\text { of project leader, name of affiliation, summary } \\
\text { of duplication project) }\end{array}$ \\
\hline
\end{tabular}

Fig. 7 Blockchain structure

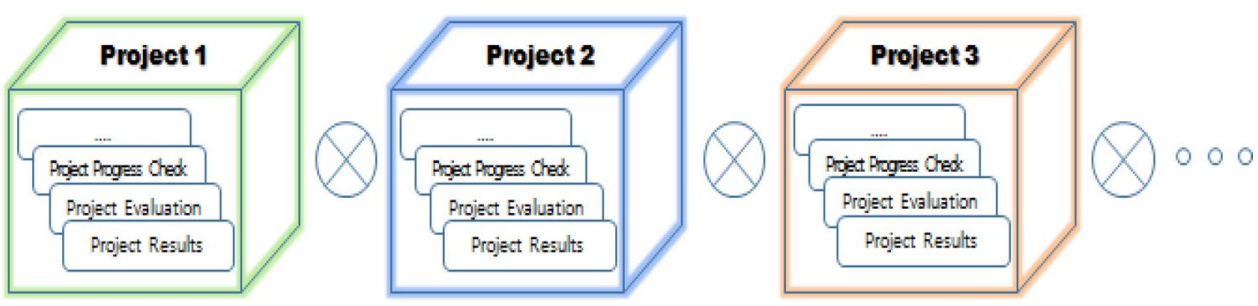


When the details of the project change, the details of the project, and duplicate project results find, performers are raised to conduct a duplication review. SuperUser has the right to create blocks when duplication of projects and project results raise. In order to proceed with block generation, agreement between SuperUser must be made. When an agreement make between SuperUsers, a block create and the duplication information is stored in the storage.

The distributed ledger uses transaction information as input values and calculates the transaction hash values using such values, as well as the SHA256 hash function (Lim et al. 2018; Pappalardo et al. 2018; Distributed ledger wiki 2018). In this paper, the transaction means the project and the ledger means the project details. The validity of the information is determined by calculating the Merkle Root values of the transaction hash values and comparing them with the Merkle Root values in the block header in order to check if they are identical. When duplication suspecte during project review, the user can inform other users of project duplication for creating a new block. When a new block create, the SuperUser determines the creation of the new block through consensus.

Figure 8 shows the entire sharing structure for sharing projects and results. The blockchain is a structure that has a link to the block immediately following, starting with the Genesis block. The blockchain has distributed storage and management across nodes. The block contains transaction information, specifically, project identification number, classification name, project name, keywords, project summary, project progress summary, and project results, which are the key elements for project duplication review. The blockchain, which is an aggregate of blocks, is a huge distributed ledger that contains all of the transaction information. In this paper, we form a sharing transaction and a duplication transaction. Each structure designe in terms of storage and management for the purpose of data sharing.

\subsubsection{Building of transaction}

Figure 9 shows the structure of the distributed ledger for the transactions that are divided into sharing, checking, and duplication transaction (Kim and Jeong 2018). Sharing and checking transactions consist of project identification number, classification name, project name, keywords, project summary, projects results, and ledger time. The duplication transaction consists of project identification number, project summary, projects results, duplication verification, and ledger time. The ledger classify according to the characteristics of the shared data. The construction of the ledgers for transaction use the Mercury tree to increase the efficiency of data storage. One of the factors affecting the performance of the blockchain system is
Fig. 8 Proposed TIP-platform block structure

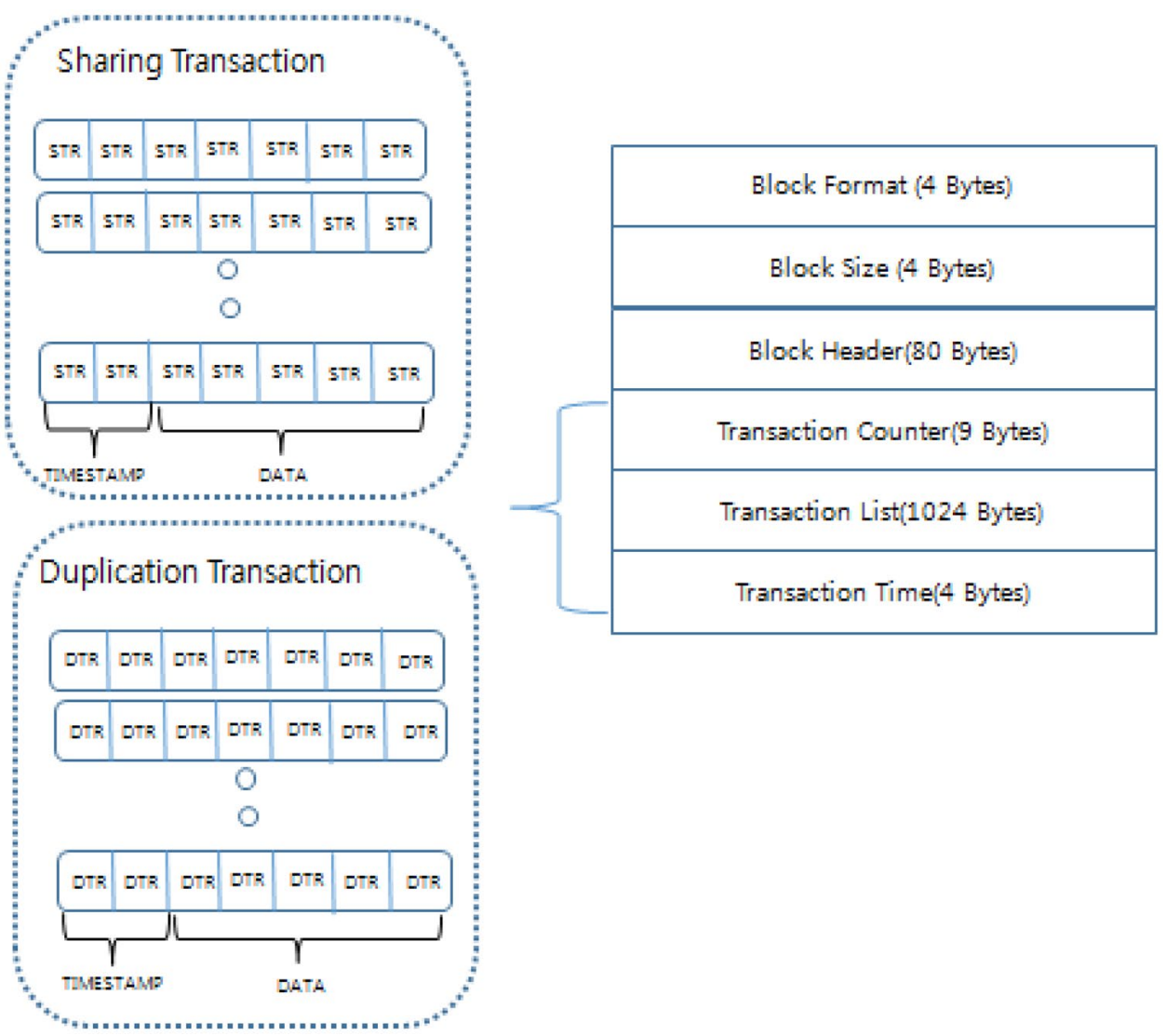


Fig. 9 Ledger structure for transactions

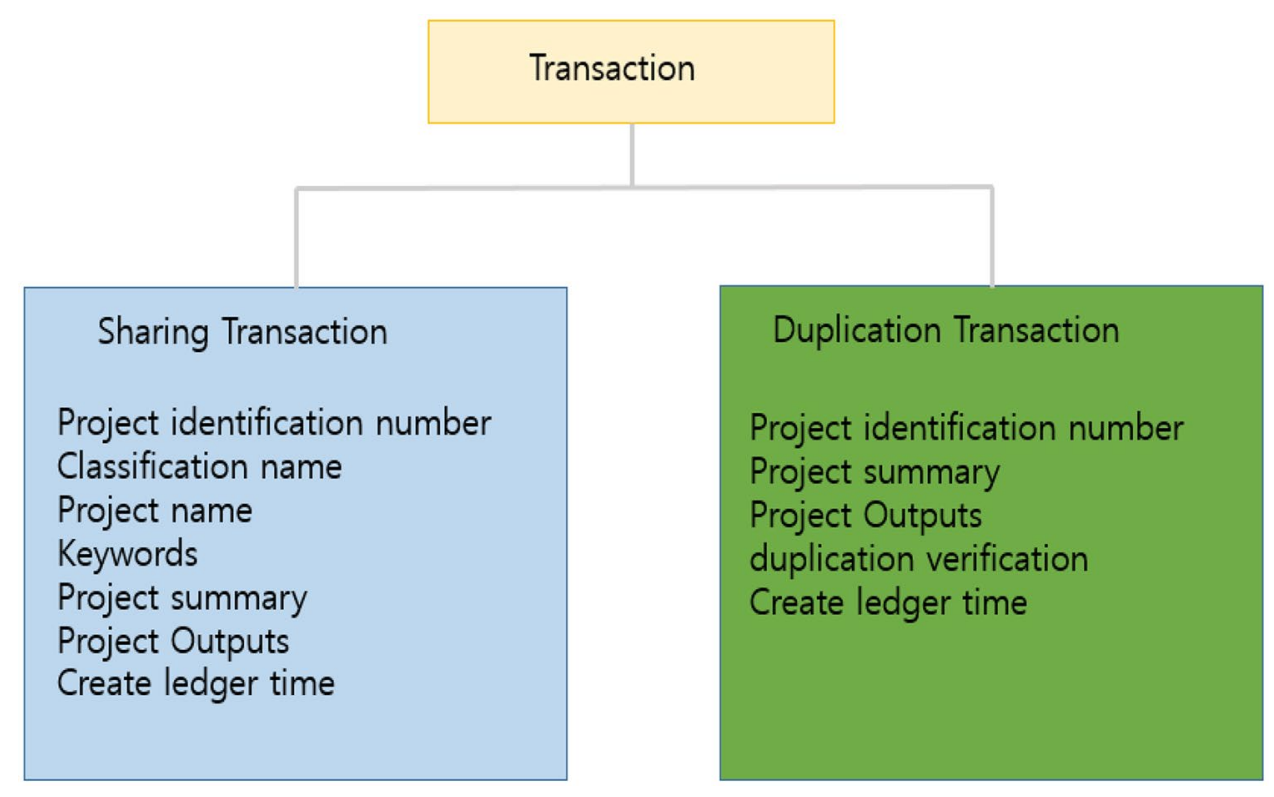

the consensus algorithm (Ongaro and Ousterhout 2014; Nguyen and Kim 2018). Many consensus algorithms allow many nodes to contain identical information, but they consume a large amount of energy for performance. In this paper, we employ a consortium blockchain method, which is one of the methods of private blockchain. Only the SuperUser (project manager or department official) agrees with and approves blockchain generation.

\subsubsection{Proposed consensus algorithm}

One of the factors affecting the performance of the block chain system is the consensus algorithm. A new node create through the consensus algorithm and many nodes can have the same information. However, if many nodes participate in the consensus to give fairness to the agreement, it can generate a lot of energy consumption in terms of performance. Recently, various consensual algorithms such as POS(Proof and Stake) and DPOS(Delegated Proof of Stake) have been developed to complement these aspects. However, there are no perfect algorithms among the algorithms. To overcome the weaknesses of existing algorithms, this paper complements the problem of excessive energy use without damaging the fairness problem. In this paper, a new concept of consensus algorithm is proposed. In this paper, Consensus algorithm requires $100 \%$ consent from the superuser who can create the block. The blocks created can provide the same information to all users. For example, if new project information change or generate by malicious intent, problems arise from the planning stage of the national $R \& D$ project with duplicate project planning. To prevent this from happening, superusers creating the block should generate $100 \%$ agreed and trusted information (Mohammadi et al. 2019).

And this algorithm features atomicity and consistency. The atomicity and consistency of this algorithm can increase reliability (Lee and Yoon 2019).

- Atomic A block is created only when $100 \%$ of the superusers agree. However, if the agreement do not reached in the middle, the block will delete.

- Consistency If the agreement is successful, the transaction information will be updated. This information provides the same information on any node.

In this paper, A consensus algorithm is proposed to reflect the characteristics of the national $R \& D$ project.

\section{Conclusions}

We proposed the TIP-Platform in order to share project information, project results, and duplication project information on the basis of blockchain technology. The TIP-Platform gives users three levels of authority, so that only the SuperUse can create blocks of information, in order to restrict the sharing of information. In addition, we proposed the structure of the transaction to reflect the characteristics of the national $R \& D$ project. Transactions are classified according to the characteristics of the information to form a ledger. In order to build ledgers, TIPPlatform uses the Mercury tree to increase the efficiency of data storage. For the process, TIP-Platform constructed 
a salable and lightweight blockchain. The agreement process has tried to minimize the waste of computer energy by employing a consortium method. Therefore, it is possible to not only share national $R \& D$ project information but also generate effective information through the TIPPlatform. In the future, we will continue to study the TIPPlatform in consideration of performance, openness, and security, and develop it as a superior platform.

Acknowledgements This research was supported by Basic Science Research Program through the National Research Foundation of Korea (NRF) funded by the Ministry of Education (2018R1D1A1B07047112).

Open Access This article is distributed under the terms of the Creative Commons Attribution 4.0 International License (http://creativeco mmons.org/licenses/by/4.0/), which permits unrestricted use, distribution, and reproduction in any medium, provided you give appropriate credit to the original author(s) and the source, provide a link to the Creative Commons license, and indicate if changes were made.

\section{References}

http://www.bloter.net/archives/261939 (2018)

https://www.bigchaindb.com (2018)

https://www.ibm.com/cloud-computing/bluemix/ko/architecture (2018)

https://www.ibm.com/cloud-computing/bluemix/ko/watson (2018)

https://www.media.mit.edu/projects/enigma (2018)

Analysis of National R\&D Project Survey Results for 2017 (2018)

National Science and Technology Advisory Council. https://www. kistep.re.kr/c3/sub2_5.jsp

Bitcoinwiki (2018) https://en.bitcoin.it/wiki/Block_hashing_algorithm BitFury Group (2015) Proof of stake versus proof of work. White Paper. https://bitfury.com/content/downloads/pos-vs-pow-1.0.2.pdf

Distributed ledger wiki (2018) https://en.wikipedia.org/wiki/Distribute d_ledger. Accessed 10 Sept 2018

Jun MS (2018) Blockchain government-a next form of infrastructure for the twenty-first century. J Open Innov Technol Mark Complex $4: 7$
Kakavand H (2017) The blockchain revolution. DLP Piper, London

Kim HW, Jeong YS (2018) Secure authentication-management humancentric scheme for trusting personal resource information on mobile cloud computing with blockchain. Hum Cent Comput Inf Sci 8:11

Kim J, Jeong MH, Kim JM, Yoo Y (2006) Block chain primer. https:// assets.korbit.co.kr/docs/Korbit-White_Paper-Block_Chain_Prime r-1.pdf

Kwak H (2017) Industrial trend and patent trend of blockchain technology. Korea Knowledge Industry Institute, Korea

Lee Eunhee, Yoon Youngik (2019) Project management model based on consistency strategy for blockchain platform. IEEE Comput Soc SERA 2019:38-44

Lim JC, Yoo HG, Kwak JY, Kim SM (2018) Blockchain and consensus algorithm. 2018 electronics and telecommunication trends. https://ettrends.etri.re.kr/ettrends/169/0905169005/. Accessed 1 Sept 2018

Mohammadi V, Rahmani AM, Darwesh AS, Sahafi A (2019) Trustbased recommendation systems in Internet of Things: a systematic literature review. Hum Cent Comput Inf Sci 2019:9-21

Nguyen GT, Kim K (2018) A survey about consensus algorithms used in blockchain. J Inf Process Syst 14(1):101-128

Ongaro D, Ousterhout JK (2014) In search of an understandable consensus algorithm. USENX Annu. Technical Conf, Philadephia, pp 305-319

Pappalardo G, Matteo T, Caldarelli G, Aste T (2018) Blockchain inefficiency in the Bitcoin peers network. EPJ Data Sci 7:30

Singh A, Chatterjee K (2018) Trust based access control model for securing electronic healthcare system. J Ambient Intell Hum Comput 1:1-19

Singh A, Chatterjee K (2019) An adaptive mutual trust based access control model for electronic healthcare system. J Ambient Intell Hum Comput 1:1-20

Swan M (2015) Blockchain, pp 27-31

Tapscott D, Tapscott A (2017) Blockchain revolution. Brilliance Audio, Grand Haven, pp 218-264

Publisher's Note Springer Nature remains neutral with regard to jurisdictional claims in published maps and institutional affiliations. 